\title{
Comparación de indicadores ambientales en tres casos de agricultura: tradicional, orgánica y convencional
}

\author{
Doris Guilcamaigua Paztuñal* \\ Edwin Chancusig Espín**
}

\begin{abstract}
RESUMEN

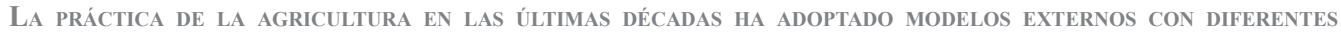
IMPACTOS AMBIENTALES, LO QUE LLEVA A PLANTEAR ALTERNATIVAS AGRíCOLAS QUE SEAN SUSTENTABLES. EN ESTE ESTUdio SE ANALIZA LA SUSTENTABILIDAD DESDE LA PERSPECTIVA AMBIENTAL DE TRES AGROECOSISTEMAS CON DIFERENTES PROPUESTAS DE PRODUCCIÓN (TRADICIONAL, ORGÁNICO Y CONVENCIONAL), UBICADOS EN LA PROVINCIA de Chimborazo, Ecuador. La metodología se basa en la caracterización de sistemas, el Cálculo de 7

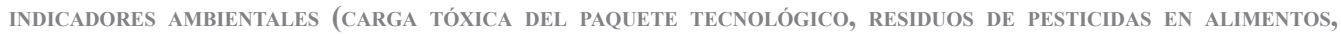

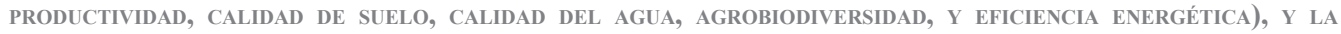
VALORACIÓN DE LA SUSTENTABILIDAD AMBIENTAL.
\end{abstract}

Palabras claves: agroecosistemas - sustentabilidad - agrobiodiversidad - Chimborazo - indicadores AMBIENTALES - TÉCNICAS ANCESTRALES.

\section{Abstract}

THE PRACTICE OF AGRICULTURE IN THE LAST DECADES HAS ADOPTED EXTERNAL MODELS WITH DIFFERENT ENVIRONMENTAL IMPACTS, WHICH LEADS TO THE PROPOSAL OF SUSTAINABLE AGRICULTURAL ALTERNATIVES. ThIS STUDY ANALYZES SUSTAINABILITY FROM THE ENVIRONMENTAL PERSPECTIVE OF THREE AGROECOSYSTEMS WITH DIFFERENT PRODUCTION proposals (TRAditional, organic AND CONVENTIONAL), LOCATEd In THE PRovince of Chimborazo, Ecuador. THE METHODOLOGY IS BASED ON THE CHARACTERIZATION OF SYSTEMS, THE CALCULATION OF SEVEN ENVIRONMENTAL INDICATORS (TOXIC LOAD OF THE TECHNOLOGICAL PACKAGE, PESTICIDE RESIDUES IN FOOD, PRODUCTIVITY, SOIL QUALITY, WATER QUALITY, AGROBIODIVERSITY, AND ENERGY EFFICIENCY), AND THE ENVIRONMENTAL SUSTAINABILITY.

Keywords: Agroecosystems - SUSTAINABILITY - AGROBIODIVERSITY - ChIMborazo - ENVIRONMENTAL INDICATORS - ANCESTRAL TECHNIQUES.

* Magister en Protección Ambiental. Universidad de las Fuerzas Armadas, Departamento de Energía y Mecánica, Docente. Correo electrónico: deguilcamaigua@espe.edu.ec

** Magister en Agroecología y Desarrollo Sustentable. Universidad Técnica de Cotopaxi, Unidad de Ciencias Agropecuarias y Recursos Naturales. Correo electrónico: edwin.chancusig@utc.edu.ec. 


\section{Introducción}

T a agricultura sustentable hace hincapié en los sistemas de producción de alimentos, que sean rentables, ecológicamente racionales y eficientes en el uso de energía, además de mejorar la calidad de vida de los agricultores y consumidores (Menalled et al., 2008: 1). Por lo tanto, la producción mundial de alimentos en la actualidad debe cumplir con el doble reto de alimentar a una población creciente y a la vez disminuir los impactos ambientales que se puedan generar (Seufert et al., 2012: 1). Está claro que no hay más remedio que producir más con menos; "la sostenibilidad ambiental en la agricultura ya no se trata de una opción sino de un imperativo" (Hamuda y Patkó, 2010: 87).

En Ecuador, estos retos aún no son abordados del todo, la toma de decisiones en el campo de la agricultura centra su énfasis en variables económicas ligadas a la productividad en el corto plazo, lo que ha llevado a la adopción de modernas tecnologías de carácter intensivo. Pocas son las evaluaciones y estudios que muestran los riesgos de la aplicación de éstas propuestas tecnológicas y sus efectos en el suelo, agua, biodiversidad, alimentos y productividad de los agroecosistemas. La especialización de las unidades de producción ha llevado a la imagen que la agricultura es un milagro moderno de la producción de alimentos. Además, "los impactos ambientales de los desequilibrios ecológicos se han asociado con la intensificación de producción de alimentos" (Ibíd.). Esta situación lleva a cuestionar entonces los objetivos, las políticas y las tecnologías que se aplican en los sistemas de producción.

"En sistemas convencionales ligados a tecnologías provenientes de la llamada Revolución Verde (RV), la instalación de fincas o potreros generalmente se realiza a costa de la integridad de los ecosistemas o utilizando monocultivos e insumos de alta demanda energética o de altos niveles de emisión de Gases de Efecto Invernadero" (Córdova y León, 2013: 22).

En contraste, en sistemas tradicionales, "establecidos en base a un conocimiento técnicoempírico que la cultura campesina, indígena e indo- mestiza ha heredado por generaciones, ha alimentado con la observación, recolección y permanente reflexión sobre los fenómenos de la producción" (Sánchez et al., 2015: 2), la tierra se trabaja con técnicas e insumos del medio y está adaptada a las condiciones locales.

En los sistemas orgánicos, "un sistema productivo que propone evitar e incluso excluir totalmente los fertilizantes y pesticidas sintéticos de la producción agrícola; la producción se realiza en lo posible reemplazando las fuentes externas tales como substancias químicas y combustibles adquiridos comercialmente, por recursos que se obtienen dentro del mismo predio o en sus alrededores" (Altieri, 1999: 160).

Comprender las características de manejo y las lógicas en el uso de las diferentes técnicas en los sistemas de producción, así como sus efectos en el ambiente, podría aportar al diseño de agroecosistemas sustentables desde la perspectiva ecológica, a la generación de propuestas para la mitigación de impactos negativos en la agricultura, al diseño de sistemas con uso eficiente de energía, que a su vez produzcan alimentos sanos y seguros. Este trabajo indaga las posibilidades de producción de tres tipos de pequeños agricultores: convencional, tradicional y orgánico, en la zona central de la serranía ecuatoriana, dedicados al cultivo de hortalizas destinadas al autoconsumo y venta de excedentes, y los evalúa desde una perspectiva ambiental.

Aun cuando la dimensión ambiental es solo una parte de la integralidad de un agroecosistema junto a los aspectos social y económico, se acepta que la salud del ecosistema es la base fundamental para la producción de alimentos en un territorio finito. "Para la dimensión ambiental, se tienen grandes listados de variables a diversos niveles: nacional (INEGI, 2000; DEFRA, 2007), por bloques de países como América Latina (CIAT-UNEP, 1998), a nivel internacional (IISD, 2007b; ONU, 2007), o a nivel de manejo especializado para un sistema 
de producción o manejo de tierra (FAO, 1993)" (Peña-Olvera et al., 2008: 276). A nivel local, estudios como el de Garcés (2016) aportan con metodologías de valoración de los sistemas agrícolas desde una perspectiva multidimensional. En el presente estudio se plantea el uso de indicadores ambientales, que sintetizan un conjunto amplio de variables en un solo dato o cifra (ver tabla 1), para finalmente evaluar la sostenibilidad ambiental de tres agroecosistemas en función de la propuesta tecnológica utilizada.

\section{Materiales y métodos}

El estudio se realizó en los cantones Colta, Guamote y Riobamba (Chimborazo, Ecuador), comparando las características ambientales generadas por las prácticas utilizadas por los agricultores para la producción agropecuaria, en tres unidades productivas agrícolas UPA ${ }^{1}$ o agroecosistemas diferentes (tradicional, orgánico y convencional). Los tres cantones se ubican en la provincia de Chimborazo, en la serranía central del Ecuador, a 3000 m.s.n.m., con una precipitación media anual de 694,1 m.m. concentrada en los meses de febrero, abril y noviembre; según (INAMHI, 2016: 97), la temperatura media anual es de $13,9^{\circ} \mathrm{C}$, con mínimas de $8^{\circ} \mathrm{C}$ y máximas de $21^{\circ} \mathrm{C}$; la humedad ambiental promedio es del $72 \%$. El clima es ecuatorial mesotérmico seco (Velásquez, 2016: 6). En cuanto al relieve, se trata de laderas de montaña con diferentes pendientes.

El agroecosistema tradicional (AT) funciona con una propuesta de producción desarrollada in situ, acorde a lo descrito por Sánchez y otros (2015) y Altieri (1999), y se ubica en la comunidad Achullay San Agustín (cantón Guamote). El agroecosistema orgánico (AO) presenta una propuesta con principios de producción ecológica, acorde a lo mencionado por Altieri (Ibíd) y se encuentra en el barrio Santa Teresita (cantón Guano). El agroecosistema convencional (AC) presenta características provenientes de una propuesta tecnológica de la llamada Revolución Verde, como la mencionada por Córdova y León (2013), y se ubica en el sector de la comunidad San Antonio (cantón Riobamba). Se consideran los casos seleccionados, representativos de su tipo en la zona, en base a referencias proporcionadas por las instituciones de desarrollo rural Heifer Ecuador ${ }^{2}$, FOIJAG $^{3}$ y CEDEIN ${ }^{4}$, quienes trabajan en el área en un promedio de dos décadas.

Metodológicamente este estudio se basó en el Análisis de Sistemas descrito en Ruíz y Oregui (2016: 4), con un diseño Ex-post facto retrospectivo, tres tratamientos (AC, AO, AT) y un muestreo razonado (no aleatorio). La información bibliográfica necesaria se obtuvo a través de búsquedas en Google y revistas especializadas; los datos de campo se obtuvieron mediante recorridos en la zona, observación participante en las unidades productivas, entrevistas a familias, análisis de muestras en laboratorio y estudios de caso. Los datos obtenidos se organizaron en una base de datos computacional con el programa Microsoft Excel y fueron procesados mediante el uso de indicadores ambientales para cada uno de los agroecosistemas. El método para cada el cálculo de cada indicador se describe a continuación:

Índice de Carga Tóxica del Paquete Tecnológico, según Alvarez y Bustamante (2006: 162163): a. Acopio de información de insumos utilizados en un ciclo de cultivo. b. Lectura de medidas de toxicidad (DL 50, principio activo) en hojas de seguridad de insumos agroquímicos.

1 "Unidad de Producción Agrícola.- La Unidad Productiva Agrícola (UPA) "corresponde a una extensión de tierra de $500 \mathrm{~m} 2$ o más, dedicada total o parcialmente a la producción agropecuaria, considerada como una unidad económica, que desarrolla su actividad bajo una dirección o gerencia única" (INEC, 2013: 1).

2 Organización No Gubernamental ecuatoriana de desarrollo rural, sin fines de lucro, vinculada a Heifer International (Heifer, 2016).

3 Federación de Organizaciones Indígenas y Campesinas Jatun Ayllu Cabildo Guamote, es una organización de segundo grado.

4 Centro de Desarrollo Indígena (Comité Ecuménico de Proyectos, 2016). 
c. Cálculo de la Potencia letal mediante Ec. 1; d. Cálculo de Carga tóxica del paquete tecnológico por unidad de superficie, mediante sumatoria de datos de Potencia Letal.

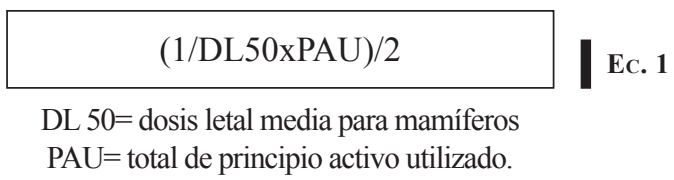

Residuos de pesticidas en alimentos, con protocolos descrito por AGROCALIDAD (s/f.: 28) e INEN $(2013,9)$ y Límites Máximos de Residuos (LMR) según FAO y OMS (2016: 1): a. Selección de parcela representativa por UPA; b. Muestreo simple aleatorio (obtención de muestra conformada por 5 submuestras recolectadas en zigzag, hasta completar $2 \mathrm{~kg}$ de cultivo) al finalizar el ciclo de producción. c. Determinación de familias de pesticidas utilizados, mediante encuesta y revisión de etiquetas y hojas de seguridad de agroquímicos; d. Análisis de muestras en laboratorio especializado en función de familias de pesticidas utilizados (Organoclorados, Organofosforados, Carbamatos) con el método de cromatografía de gases; e. Evaluación de resultados en base LMR del Codex alimentarius de la FAO.

Productividad: Biomasa por hectárea, según (Gliessman, 2002: 311): a. Selección de 1 parcela representativa por UPA; b. Muestreo al azar en X para la ubicación de unidades de superficie de $1 \mathrm{~m}^{2}$; c. Cosecha de cultivo en pie y obtención del peso total. b. Obtención de valores de porcentaje de humedad de cada especie de cultivo, y cálculo del peso seco por unidad de superficie (ton/ha); d. Cálculo el Índice de Productividad (IP), con la Ec. 2.

$$
\begin{array}{c|}
\text { IP }=\frac{\text { Total de biomasa acumulada en el sistema* }}{\text { Productividad Primaria Neta }(\mathrm{PPN})} \\
\mathrm{PPN}=\text { cantidad de energía que queda almacenada en los productores y disponible para } \\
\text { consumidores en forma de biomasa, en un ecosistema }{ }^{5}
\end{array}
$$

Calidad del suelo, según Altieri (1999: 282) y Garcés (2011: 34): a. Selección de unidades de muestreo de suelos: 1 parcela representativa por UPA y 1 parcela por sistema natural (espacios con vegetación silvestre o bosque) en cada UPA; b. Muestreo con varias repeticiones (30 submuestreos en cada tratamiento y 10 submuestreos en cada sistema natural, tomados aleatoriamente, hasta completar $1 \mathrm{Kg}$.) (Buduba, 2016: 10); c. Análisis de parámetros: materia orgánica, $\mathrm{pH}$, textura, macro y microelementos $(\mathrm{N}, \mathrm{P}, \mathrm{K}, \mathrm{Ca}, \mathrm{Mg}, \mathrm{Fe}$, $\mathrm{Mn}, \mathrm{Cu}, \mathrm{Zn}, \mathrm{B}, \mathrm{S})$, en laboratorio; d. Evaluación de resultados en base a normas y criterios establecidos en Potash \& Phosphate Institute (1997); e. Análisis comparativo entre datos de unidades de muestreo (suelo de cultivo vs suelo de vegetación natural), bajo el enfoque cronosecuencial ${ }^{6}$.

5 Dato corresponde al valor promedio de la Productividad Primaria Neta Aérea en relación a condiciones edáficas y disponibilidad de agua en el ecosistema terrestre Bosques y arbustales templados y templados - fríos; con total capacidad productiva del suelo. Referencia bibliográfica en: www.fcnym.unlp.edu.ar/catedras/ecocomunidades/ Gallopin_PPN.pdf(Gómez y Gallopín, 2016: 5).

6 El enfoque cronosecuencial demuestra cambios en las propiedades del suelo bajo un tipo particular de sistema de uso del suelo. Los niveles originales se usan como referencia para investigar las propiedades. Es mucho más útil analizar al mismo tiempo las propiedades de manera comparativa con otros sistemas de uso del suelo, por ejemplo con bosques naturales en el mismo periodo, según Poels y Stoorvogel en 1997 (Garcés, 2011: 44). 
Calidad del agua, según García (2016: 3) y Gómez (2016: 5): a. Muestreo puntual en sitio de suministro o descarga de agua en base a normas $\mathrm{INEN}^{7}$; b. Análisis de laboratorio de parámetros físicos - químico y microbiológico $(\mathrm{pH}$, coliformes, sólidos disueltos, nitritos, nitratos y salinidad); c. Análisis de resultados en base a normas y criterios establecidos en el Texto Unificado de Legislación Secundaria del Ministerio del Ambiente, TULSMA ${ }^{8}$.

Agrobiodiversidad, según Moreno (2001: 29-44): a. Inventario de la población de especies y su abundancia, dentro de los límites geofísicos establecidos; b. Evaluación de Biodiversidad Alfa, mediante cálculo de índices: Margalef (para riqueza de especies), con Ec.3, y Simpson (para estructura), con Ec.4; c) Uso de escalas definidas para interpretación de datos.

$$
D_{M g}=\frac{S-1}{\operatorname{Ln~N}} \text { Ec. } 3
$$

Donde $\mathrm{S}=$ número total de especies y $\mathrm{N}=$ número total de individuos observados.

$$
\mathrm{D} S i=\Sigma p i^{2}
$$

EC. 4

Donde $\mathrm{pi}$ = abundancia proporcional de la iésima especie; representa la probabilidad de que un individuo de la especie i esté presente en la muestra; $\mathrm{pi}=\mathrm{ni} / \mathrm{N}$; ni= número de individuos de la especie $i ; \mathrm{N}=$ número total de individuos para todas las especies de la comunidad. Al ser su valor inverso a la equidad, la diversidad puede calcularse como 1 - DSi.

Eficiencia energética del sistema, según Risoud (2000: 2): a. Acopio de información sobre insumos agrícolas utilizados durante el ciclo de producción del producto; b. Cálculo de valores de energía no renovable utilizada para producir dicho producto, en base a tablas de valores de eficiencia energética de insumos agrícolas; b. Cálculo del Índice de Risoud, con la Ec. 5:

$$
\text { EE }(\text { Eficiencia Energética })=\frac{\text { Valor energético del producto }}{\begin{array}{c}
\text { Energía no renovable utilizada para } \\
\text { producir dicho producto }
\end{array}}
$$

Donde Valor energético del producto $=$ biomasa $^{9}$; Energía no renovable utilizada durante los procesos de producción = energía indirecta (extracción de las materias primas, fabricación del producto y transporte) + energía directa utilizada dentro del sistema (electricidad, gasolina, aceites, químicos sintéticos). Lectura e interpretación de resultados.

7 Ecuador, Instituto Ecuatoriano de Normalización. 1998. "NTE INEN 2169: Agua. Calidad del agua. Muestreo. Manejo y conservación de muestras -ec.nte.2169.1998" https://law.resource.org/pub/ec/ibr/ec.nte.2169.1998.pdf.

8 Ecuador, Ministerio del Ambiente. 2014, "Norma de Calidad Ambiental y de Descarga de Efluentes: Recursos Agua", en: http://www.industrias.ec/archivos/CIG/file/CARTELERA/Reforma\%20Anexo $\% 2028 \% 20 \mathrm{feb} \% 20$ 2014\%20FINAL.

9 Valor energético de hortalizas y cultivos (Rozano, 2016). 
Comparación de Tratamientos en base al Marco Referencial para la Evaluación de la Sustentabilidad, MESMIS (López et al., 2001): a. Determinación de puntos críticos o procesos que hacen peligrar o refuerzan la sostenibilidad del sistema, en base a la pregunta: ¿Qué hace que el sistema sea vulnerable?; b. Valoración de cada indicador en una escala de 1 a 5 , en donde 1 representa menor aporte para la sustentabilidad ambiental (muy bajo) y 5 la máxima (muy alto), como se indica en la tabla 1; c. Diagramación tipo AMIBA que muestra cualitativamente, lo que se ha logrado del objetivo según cada indicador, dando el porcentaje del valor real con respecto al valor ideal.

\begin{tabular}{|c|c|c|c|c|}
\hline Punto Crítico & Indicador & Concepto & Parámetros & Valor \\
\hline \multirow[b]{2}{*}{ Carga tóxica alta } & \multirow[b]{2}{*}{$\begin{array}{l}\text { Carga tóxica } \\
\text { del paquete } \\
\text { tecnológico }\end{array}$} & \multirow{2}{*}{$\begin{array}{l}\text { "Letalidad en mamíferos } \\
\text { de los productos } \\
\text { agroquímicos usados en } \\
\text { cada uno de los paquetes } \\
\text { tecnológicos" (Álvarez y } \\
\text { Bustamante, 2006: } 162 \\
\text { - 163). }\end{array}$} & $\begin{array}{l}\text { Nula: Uso de plaguicidas } \\
\text { naturales }\end{array}$ & 5 \\
\hline & & & $\begin{array}{l}\text { Extremadamente alta: } \\
\text { Uso de plaguicidas con } \\
\text { categoría de toxicidad } \\
\text { I, II, III y IV; tóxico a } \\
\text { extremadamente tóxico. }\end{array}$ & 1 \\
\hline \multirow{2}{*}{$\begin{array}{l}\text { Residuos de } \\
\text { plaguicidas } \\
\text { presentes }\end{array}$} & \multirow{2}{*}{$\begin{array}{l}\text { Residuos de } \\
\text { pesticidas en } \\
\text { alimentos }\end{array}$} & \multirow{2}{*}{$\begin{array}{l}\text { "Cualquier sustancia } \\
\text { especificada presente } \\
\text { en alimentos, productos } \\
\text { agrícolas o alimentos } \\
\text { para animales como } \\
\text { consecuencia del uso de } \\
\text { un plaguicida" (FAO y } \\
\text { OMS, 2016: 1). }\end{array}$} & $\begin{array}{l}\text { Ausencia de residuos por } \\
\text { cero uso de plaguicidas }\end{array}$ & 5 \\
\hline & & & $\begin{array}{l}\text { Residuos de plaguicidas } \\
\text { en alimentos }\end{array}$ & 1 \\
\hline \multirow{5}{*}{$\begin{array}{l}\text { Baja } \\
\text { productividad }\end{array}$} & \multirow{5}{*}{$\begin{array}{l}\text { Productividad: } \\
\text { biomasa por } \\
\text { hectárea }\end{array}$} & \multirow{5}{*}{$\begin{array}{l}\text { "Cantidad de biomasa } \\
\text { invertida en el producto } \\
\text { cosechado, en relación } \\
\text { con la cantidad total } \\
\text { de biomasa en pie } \\
\text { presente en el resto del } \\
\text { sistema. Capacidad del } \\
\text { agroecosistema para } \\
\text { brindar un cierto nivel } \\
\text { de bienes y servicios" } \\
\text { (Gliessman, 2002: } 311 \text { ). }\end{array}$} & IP mayor a 2 & 5 \\
\hline & & & IP de 1,6 a 2,0 & 4 \\
\hline & & & IP de 1,1 a 1,5 & 3 \\
\hline & & & IP de 0,6 a 1 & 2 \\
\hline & & & IP menor a 0,5 & 1 \\
\hline
\end{tabular}




\begin{tabular}{|c|c|c|c|c|}
\hline \multirow{5}{*}{$\begin{array}{l}\text { Decrecimientos } \\
\text { en materia } \\
\text { orgánica MO y } \\
\text { nutrientes en } \\
\text { suelos de cultivo }\end{array}$} & \multirow{5}{*}{ Calidad del suelo } & \multirow{5}{*}{$\begin{array}{l}\text { "Propiedades químicas, } \\
\text { físicas y biológicas. La } \\
\text { materia orgánica (MO) } \\
\text { influye en casi todas las } \\
\text { propiedades importantes } \\
\text { que contribuyen a la } \\
\text { calidad del suelo". } \\
\text { (Altieri, 1999: 282) }\end{array}$} & $\begin{array}{l}\text { Incremento } \\
\text { M.O, incremento } \\
\text { macronutrientes }\end{array}$ & 5 \\
\hline & & & $\begin{array}{l}\text { Incremento M.O, descenso } \\
\text { macronutrientes }\end{array}$ & 4 \\
\hline & & & $\begin{array}{l}\text { Descenso M.O, } \\
\text { incremento } \\
\text { macronutrientes }\end{array}$ & 3 \\
\hline & & & $\begin{array}{l}\text { Descenso M.O, descenso } \\
\text { macronutrientes }\end{array}$ & 2 \\
\hline & & & $\begin{array}{l}\text { Descenso M.O., descenso } \\
\text { macro y micronutrientes }\end{array}$ & 1 \\
\hline \multirow[t]{2}{*}{$\begin{array}{l}\text { Agua no apta para } \\
\text { uso agropecuario }\end{array}$} & \multirow[t]{2}{*}{ Calidad del agua } & \multirow{2}{*}{$\begin{array}{l}\text { "Los criterios se basan } \\
\text { en condiciones propias } \\
\text { de cada caso. Sodicidad, } \\
\text { alcalinidad, toxicidad, } \\
\text { infiltración de agua en el } \\
\text { suelo, combinación de } \\
\text { las anteriores y efectos } \\
\text { causados por sólidos } \\
\text { en suspensión, metales } \\
\text { pesados, corrosividad" } \\
\text { (García, 2016: 3) y } \\
\text { (Gómez, 2016: 5). }\end{array}$} & $\begin{array}{l}\text { Sin restricción - No } \\
\text { supera valores máximos } \\
\text { permisibles }\end{array}$ & 5 \\
\hline & & & $\begin{array}{l}\text { Restricción - Supera } \\
\text { valores máximos } \\
\text { permisibles }\end{array}$ & 1 \\
\hline \multirow{5}{*}{$\begin{array}{l}\text { Baja riqueza en } \\
\text { agrobiodiversidad } \\
\text { (riqueza) }\end{array}$} & \multirow{5}{*}{ Agrobiodiversidad } & \multirow{5}{*}{$\begin{array}{l}\text { "Variedad y variabilidad } \\
\text { de animales, plantas y } \\
\text { microorganismos de la } \\
\text { tierra que son importantes } \\
\text { para la alimentación y la } \\
\text { agricultura y que resultan } \\
\text { de la interacción entre el } \\
\text { ambiente, los recursos } \\
\text { genéticos y el manejo de } \\
\text { los sistemas y prácticas } \\
\text { empleadas por la gente" } \\
\text { es el concepto de FAO } \\
\text { (1998), mencionado por } \\
\text { PRATEC (2004: 27) }\end{array}$} & R1 mayor a 5 & 5 \\
\hline & & & $\mathrm{R} 1 \mathrm{de} 4,1$ a 5,0 & 4 \\
\hline & & & $\mathrm{R} 1 \mathrm{de} 3,1$ a 4,0 & 3 \\
\hline & & & $\mathrm{R} 1 \mathrm{de} 2,1$ a 3,0 & 2 \\
\hline & & & R1 menor a 2 & 1 \\
\hline \multirow{5}{*}{$\begin{array}{l}\text { Uso ineficiente de } \\
\text { la energía }\end{array}$} & \multirow{5}{*}{$\begin{array}{l}\text { Eficiencia } \\
\text { energética del } \\
\text { sistema }\end{array}$} & \multirow{5}{*}{$\begin{array}{l}\text { "Eficiencia del proceso de } \\
\text { cultivo; valor energético } \\
\text { de los productos agrícolas } \\
\text { en relación con la energía } \\
\text { no renovable utilizada } \\
\text { para producirlos" } \\
\text { (Risoud, 2016: 2). }\end{array}$} & EE mayor a 0,41 & 5 \\
\hline & & & EE de 0,31 a 0,40 & 4 \\
\hline & & & EE de 0,21 a 0,30 & 3 \\
\hline & & & EE de 0,11 a 0,20 & 2 \\
\hline & & & EE 0 a 0,10 & 1 \\
\hline
\end{tabular}


El presente trabajo no podrá ser utilizado para generalizar la realidad agrícola en el país, por cuanto se trata de únicamente de tres estudios de caso. El número y tipo de indicadores fueron seleccionados en base interés de los actores vinculados al tema y la disponibilidad de métodos, datos e información. Se podría ampliar el número de indicadores.

\section{Resultados}

\section{Características generales de los agricultores}

En la tabla 2 se resumen los principales rasgos o características de orden socioeconómico de los tres agricultores que participaron en este estudio. A partir de su análisis se puede hacer las siguientes observaciones: Son familias constituidas por padres e hijos; en los casos AT y AC destaca la juventud de los agricultores cabeza de hogar; en los tres casos los hijos manifiestan deseo de continuar trabajando en las UPAs. El tiempo de residencia en la AC es mayor (20 años), se trata de una familia que inició la vida laboral en la agricultura desde edades muy tempranas. Las UPAs en los tres casos son de tenencia propia, en los AT y AC la superficie no supera las 10 ha y están en uso en el $100 \%$ de su superficie, mientras que en el AO la superficie es de 20 ha, de ella solo 4 ha bajo cultivo.

\section{TABLA 2. RASGOS SOCIALES Y ECONÓMICOS DE LOS AGROECOSISTEMAS}

\begin{tabular}{|l|c|c|c|}
\hline Rasgos sociales y económicos & AO & AT & AC \\
\hline Tiempo permanencia (años) & 8 & 11 & 20 \\
\hline Edad familia & $60-55 ; 15-11-9$ & $37-35 ; 11-5$ & $39-38 ; 19-10$ \\
\hline Tenencia tierra & Propia & Propia & Propia \\
\hline Tamaño UPA (has) & 20 & 2,36 & 6 \\
\hline Tierra cultivada & 4 & 2,36 & 6 \\
\hline Ciclos de cultivo/año & 3 & 2 & 3 \\
\hline Riego & SI - P & C - I - P & CI \\
\hline Origen semillas & CA, EE, AP & CA, EE, AP & CA, EE, AP \\
\hline Instalaciones & B & B & B \\
\hline Vías de acceso & $16 \mathrm{Km}$ & $21 \mathrm{Km}$ & $2 \mathrm{Km}$ \\
\hline Distancia a los mercados & V - A & V -A & V \\
\hline Destino de la producción & & & \\
\hline
\end{tabular}

$\mathrm{C}=$ compra $\mathrm{I}=$ intercambio $\mathrm{P}=$ producción propia

$\mathrm{CA}=$ casa $\quad \mathrm{EE}=$ energía eléctrica $\mathrm{AC}=$ alcantarillado $\mathrm{AP}=$ agua potable

$\mathrm{B}=$ buenas $\mathrm{R}=$ regulares $\mathrm{M}=$ malas

$\mathrm{V}=$ venta $\quad \mathrm{A}=$ autoconsumo

Los ciclos de cultivo en los tres casos fluctúan de 2 a 3. El AT es el único que no posee riego. Para la adquisición de semillas los agricultores recurren a diferentes modalidades, destacando que el AC es el único que solo recurre a la compra de este insumo. Los tres casos poseen instalaciones básicas (casa, energía eléctrica, y agua potable), carecen de servicio de alcantarillado y cuentan 
con buenas vías de acceso. Un rasgo relevante es el destino de la producción, los AO y AT producen para la venta y el autoconsumo, solo el AC destina su producción totalmente para la venta. Los motivos para la elección de las propuestas técnicas de manejo de las UPAs, según entrevistas, obedecen a recursos económicos a invertir, cercanía a los mercados, información sobre insumos, educación y motivaciones filosóficas de vida.

\begin{tabular}{|c|c|c|c|c|}
\hline \multicolumn{2}{|c|}{ Indicador o criterio ambiental } & AO & AT & $\mathbf{A C}$ \\
\hline \multicolumn{2}{|l|}{ Prácticas utilizadas } & Orgánicas & Tradicionales & Convencionales \\
\hline \multicolumn{2}{|c|}{$\begin{array}{l}\text { Carga tóxica del paquete tecnológico } \\
(\mathrm{kg})\end{array}$} & $\begin{array}{l}\text { Nula: No utiliza } \\
\text { plaguicidas }\end{array}$ & $\begin{array}{l}\text { Nula: No utiliza } \\
\text { plaguicidas }\end{array}$ & $\begin{array}{l}\text { Extremadamente } \\
\text { alta: } 193711,05 \\
\text { Kg mamif./ha }\end{array}$ \\
\hline \multicolumn{2}{|c|}{$\begin{array}{l}\text { Residuos de pesticidas en alimentos } \\
(\mathrm{mg} / \mathrm{Kg})\end{array}$} & $\begin{array}{l}\text { Ausente: No } \\
\text { utiliza plaguicidas }\end{array}$ & $\begin{array}{l}\text { Ausente: No } \\
\text { utiliza plaguicidas }\end{array}$ & $\begin{array}{l}\text { Presente: } 0,027 \\
\text { mg/Kg Clorpirifos } \\
\text { (Insecticida } \\
\text { Organofosforado). }\end{array}$ \\
\hline \multicolumn{2}{|c|}{ Productividad: Biomasa por hectárea } & 2,51 (Muy alta) & 2,57 (Muy alta) & 1,77 (Alta) \\
\hline \multirow[b]{2}{*}{$\begin{array}{l}\text { Calidad del suelo } \\
(\uparrow),(\downarrow),(=)^{*}\end{array}$} & Materia Orgánica & $1,81 \%(\uparrow)$ & $8,40 \%(\uparrow)$ & $1,89 \%(\downarrow)$ \\
\hline & $\begin{array}{l}\text { Micro y } \\
\text { macronutrientes } \\
\text { asimilables } \\
\text { (análisis } \\
\text { cronosecuencial) }\end{array}$ & $\begin{array}{l}\text { N, K, Ca, Zn, Cu, } \\
\mathrm{Mg}(\uparrow) \\
\mathrm{Fe}, \mathrm{P}(\downarrow) \\
\mathrm{Mn}(=)\end{array}$ & $\begin{array}{l}\mathrm{K}, \mathrm{P}, \mathrm{Zn}(\uparrow) \\
\mathrm{N}, \mathrm{Ca}, \mathrm{Fe}, \mathrm{Cu}, \\
\mathrm{Mg}(\downarrow) \\
\operatorname{Mn}(=)\end{array}$ & $\begin{array}{l}\mathrm{N}, \mathrm{P}, \mathrm{Fe}, \mathrm{Cu}, \mathrm{Mn}, \\
\mathrm{Mg}, \mathrm{Zn}(\uparrow) \\
\mathrm{K}, \mathrm{Ca}(\downarrow)\end{array}$ \\
\hline \multirow{3}{*}{ Calidad del agua } & & Apta & Apta & No apta \\
\hline & $\begin{array}{l}\text { Valores máximos } \\
\text { permisibles } \\
(\text { TULSMA, } \\
2003)^{10}\end{array}$ & No supera & No supera & $\begin{array}{l}\text { Supera en: } \\
\text { Coliformes totales } \\
(>1 \times 106 \mathrm{UFC} / 100 \\
\mathrm{mL})^{* *}\end{array}$ \\
\hline & $\begin{array}{l}\text { Valores máximos } \\
\text { permisibles } \\
\text { (TULSMA, } \\
2003)^{11}\end{array}$ & No supera & No supera & $\begin{array}{l}\text { Supera en : } \\
\text { Coliformes fecales } \\
(>1 \times 106 \text { UFC } / 100 \\
\text { mL }) \\
\text { Coliformes totales }\end{array}$ \\
\hline \multirow{2}{*}{ Agrobiodiversidad } & $\begin{array}{l}\text { Índice de Margalef } \\
\text { (riqueza) }\end{array}$ & 6,51 (Muy alta) & 4,53 (Alta) & 0,56 (Baja) \\
\hline & $\begin{array}{l}\text { Índice de Simpson } \\
\text { (distribución) }\end{array}$ & 0,90 (Alta) & 0,77 (Alta) & 0,83 (Alta) \\
\hline \multicolumn{2}{|c|}{ Eficiencia energética del sistema } & 0,22 (Media) & 0,77 (Muy alta) & 0,0030 (Muy baja) \\
\hline
\end{tabular}

* $(\uparrow),(\downarrow),(=)$ Sube, Baja, Se mantiene; análisis con respecto a suelo de bosque (enfoque cronosecuencial).

** UFC/100 ml Unidades formadoras de colonia por cada $100 \mathrm{ml}$. 


\section{Indicadores ambientales}

Se observa los valores de indicadores ambientales en la tabla 3, para AO, AT y AC. La carga tóxica del paquete tecnológico es única y extremadamente alta para el AC, siendo su valor 193711,05 Kg mamif/ha; debido a la intensiva aplicación de plaguicidas. En los AO y AT la carga tóxica es nula, por cuanto no se aplican agroquímicos. Se detectaron residuos de pesticidas (organofosforados) en muestras de coliflor del AC $(0,027 \mathrm{mg} / \mathrm{Kg}$ Clorpirifos, Insecticida Organofosforado), sin que se supere los LMR según el Codex de la FAO. Los alimentos producidos en los AT y AO están libres de plaguicidas. En productividad: biomasa por $h a$, los AO y AT y AC poseen altos índices y por lo tanto son considerados altamente productivos. En la calidad del suelo, se valoró los incrementos de MO y nutrientes del suelo de cultivo respecto al control (suelo de bosque), detectándose valores altos para el AO y AT, y valores medios para el AC.

En cuanto a la calidad del agua, en los AO y AT, el agua cumple con los parámetros del TULAS (2003), y por lo tanto es apta para uso agropecuario; no así sucede para el AC, en el que definitivamente el agua no es apta para el uso. Para agrobiodiversidad, el AO y el AT según los índices de Simpson y Margalef son altos en riqueza y distribución de especies. El AC es bajo en riqueza y alto en distribución de especies. La eficiencia energética del sistema ${ }_{2} \mathrm{EE}$ es de un nivel medio para el AO debido al uso de combustibles para la distribución de la producción. En el AT es de nivel alto, por cuanto no se usa energía no renovable en el ciclo productivo. En el AC es bajo debido al uso de combustibles, plaguicidas y fertilizantes, por tanto es menos eficiente desde el punto de vista energético.

En la tabla 4 se presentan los valores asignados a los índices ambientales en función del análisis cualitativo y cuantitativo acorde a los criterios presentados en la tabla 1. En los 7 indicadores monitoreados, el AO presenta valores muy altos en 6 y valores medios en 1 (gráfico 1), el AT valores muy altos en 5 y valores altos en 2 (gráfico 2) mientras que el AC presenta valores altos en 1 , valores medios en 1 , y valores bajos y muy bajos para el resto de indicadores (gráfico 3). A medida que los valores se acercan a los vértices del gráfico tipo "telaraña" aumenta la valoración positiva para cada una de las variables en estudio, y por lo tanto se dice que el agroecosistema se acerca a la sostenibilidad. Lo contrario sucede al acercarse los valores al centro del gráfico.

\section{TABLA 4. ASIGNACIÓN DE VALORES A ÍNDICES AMBIENTALES}

\begin{tabular}{|l|c|c|c|}
\hline Indicadores & AO & AT & AC \\
\hline Carga tóxica del paquete tecnológico & 5 & 5 & 1 \\
\hline Residuos de pesticidas en alimentos & 5 & 5 & 2 \\
\hline Productividad: Biomasa por hectárea & 5 & 4 & 4 \\
\hline Calidad del suelo & 5 & 5 & 3 \\
\hline Calidad del agua & 5 & 4 & 1 \\
\hline Agrobiodiversidad & 5 & 5 & 1 \\
\hline Eficiencia energética del sistema & 3 & & \\
\hline
\end{tabular}

10 TULSMA, 2003. Libro VI. Tabla 6. Criterios de calidad admisibles para aguas de uso agrícola.

11 TULSMA, 2003. Libro VI. Tabla 8. Criterios de calidad para aguas de uso pecuario. 


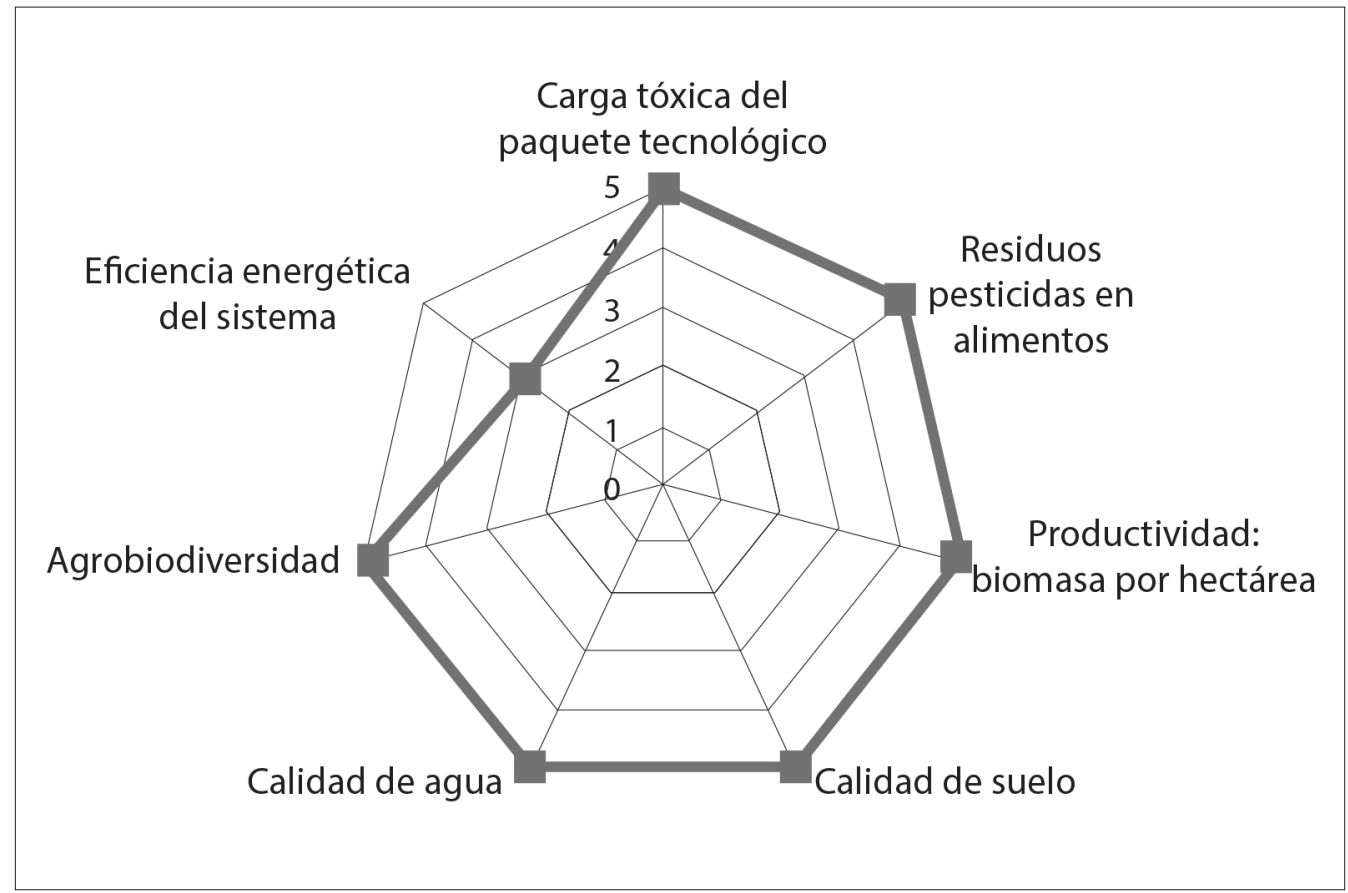

Gráfico 1. Diagrama sustentabilidad ambiental AO

Agrobiodiversidad

Carga tóxica del paquete tecnológico

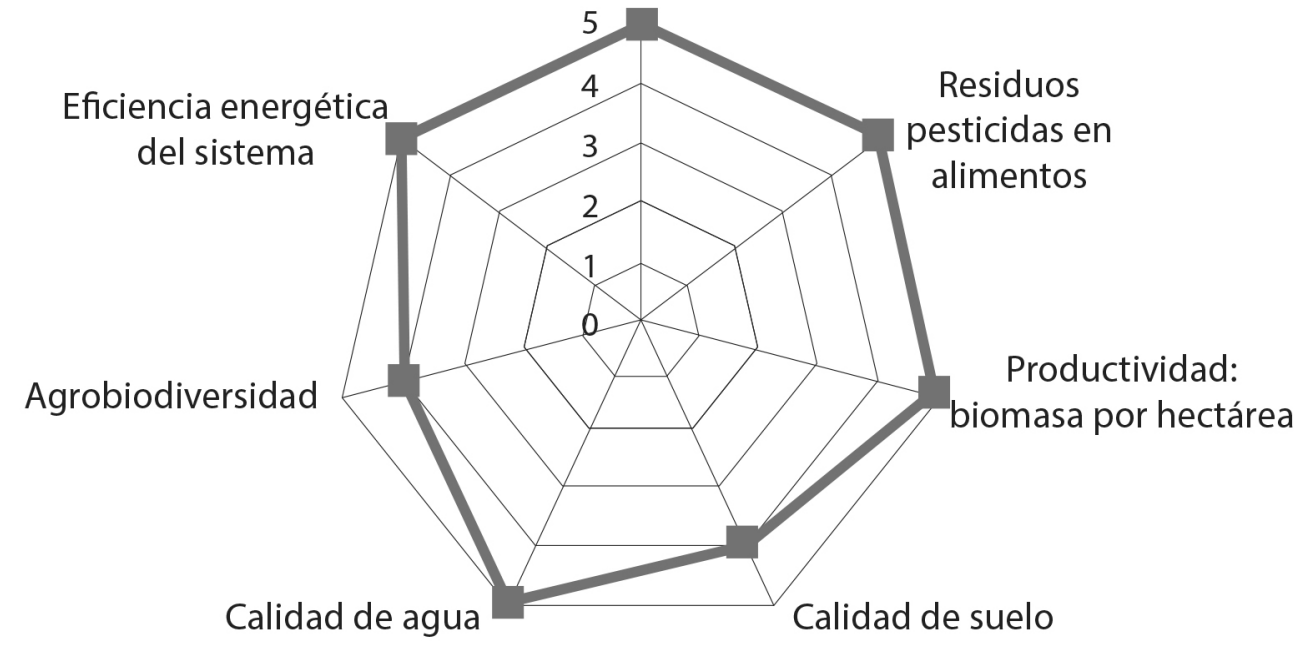

Gráfico 2. Diagrama sustentabilidad ambiental AT 


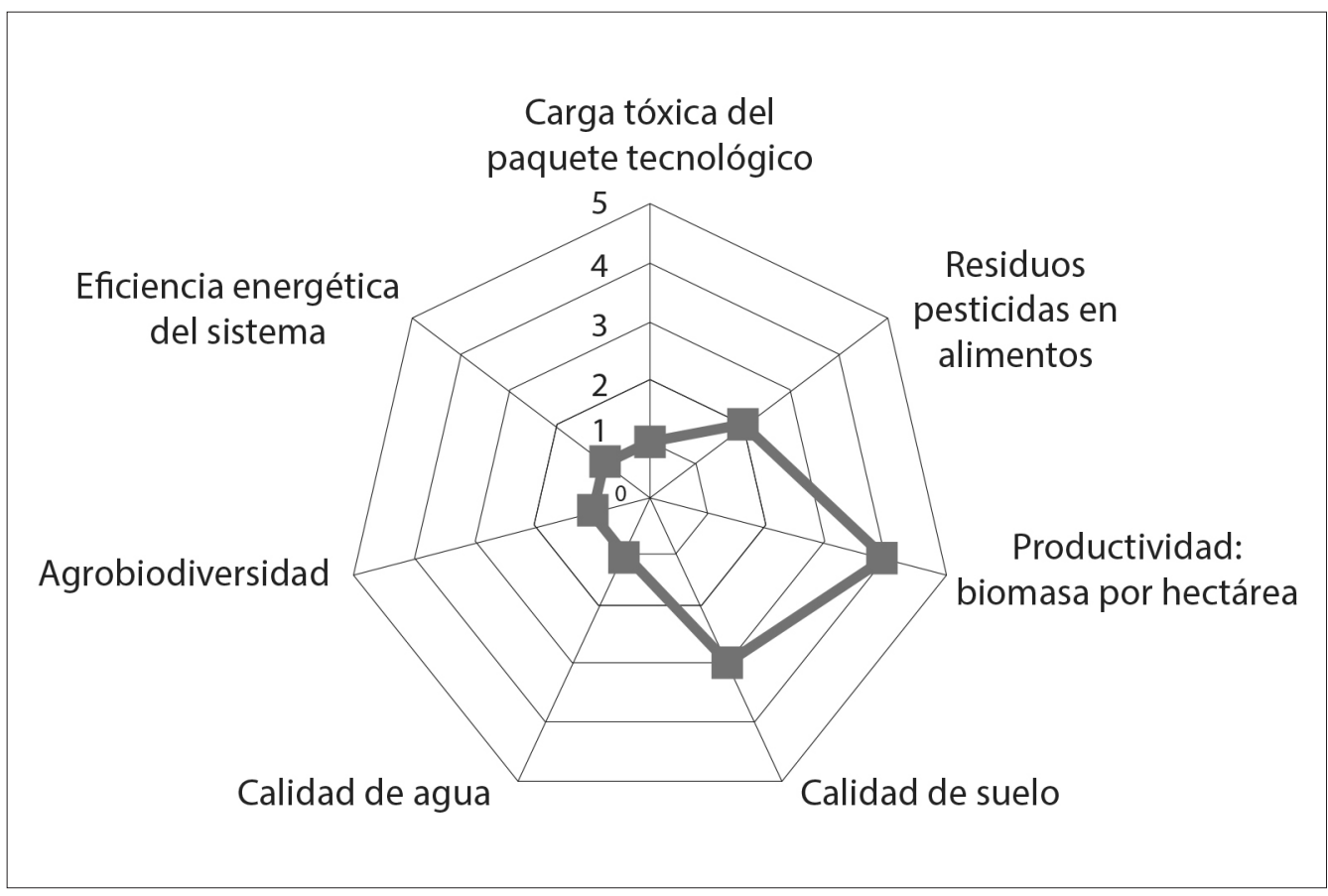

Gráfico 3. Diagrama sustentabilidad ambiental AC

\section{Discusión}

Los tres agroecosistemas mantienen diferencias notables por cuanto difieren en sus propuestas técnicas de manejo. La decisión en la elección del conjunto de técnicas obedece a factores de tipo socioeconómico (a invertir en la UPA, cercanía a los mercados, acceso a información, destino de la producción) y filosófico (elección entre obtención de dinero y bienestar familiar). En el AC el agricultor destina el $100 \%$ de la producción para la venta en el mercado, mientras que en los AO y AT los productos generados se destinan al autoconsumo y para la venta. Esta decisión podría ser influyente en las variables ambientales analizadas; así, la carga tóxica del paquete tecnológico es única y extremadamente alta $(193711,05 \mathrm{Kg}$ mamífero/ha) para el AC, debido al uso excesivo de todo tipo de plaguicidas; en este caso, se asume que la lógica de producción obedece a los estándares del mercado ("productos uniformes, limpios y de gran tamaño") y al afán de recuperar la inversión a cualquier costo (salud ambiental, salud familiar).

La cifra de $193711,05 \mathrm{~kg}$ mamif/ha es reveladora y a la vez alarmante porque muestra la cantidad de mamíferos en kg que pueden morir en el caso de ingerir por vía oral los agrotóxicos utilizados en el cultivo de hortalizas. Si se plantea un ejemplo siguiendo la metodología aplicada por Garcés (2011: 34 ) se puede dividir esta cifra para 23, $45 \mathrm{~kg}$; que representa el peso promedio de un niño de 8 años, con lo que se obtiene el número de individuos que moriría en el caso de ingerir la carga tóxica. El valor es de 8261 niños que morirían. Esta sencilla operación permite imaginar el potencial letal que un paquete de agrotóxicos tiene para destruir la vida. Se debe considerar además la forma en que son manipulados estos productos desde la comercialización, pasando por almacenamiento, uso y disposición de residuos, en los que el agricultor entra en contacto directo con las diferentes fórmulas. 
Se detecta la presencia de residuos de pesticidas $(0,027 \mathrm{mg} / \mathrm{Kg}$ Clorpirifos, Insecticida Organofosforado) en muestras de coliflor proveniente del AC. Los valores no superan los LMR establecidos en el Codex Alimentarius de la FAO, sin embargo resulta preocupante constatar que uno de los alimentos con mayor demanda en el consumo por parte de la población, posee residuos de plaguicidas. Si se proyectara el efecto acumulativo en base a la frecuencia y cantidad de productos consumidos, así como los efectos por interacción de uno y otro plaguicida, los resultados podrían ser alarmantes. Los alimentos producidos en los AO y AT al encontrarse libres de plaguicidas, estarían garantizando el acceso de sus consumidores a productos sanos y limpios.

En cuanto a la productividad: biomasa por ha, se observa que los AT y AO con Índices de Productividad, IP de 2,57 y 2,51 respectivamente son los más productivos. Esta situación puede deberse a la máxima disposición del espacio vertical y horizontal mediante prácticas propias de la propuesta técnica en uso (asociación de cultivos, presencia de hierbas silvestres, distancias mínimas de siembra) con mayor disposición para el aprovechamiento de la energía solar. En el agroecosistema convencional se tiene un IP de 1,77, considerado alto, pero relativamente menor respecto a los dos agroecosistemas en estudio complementario (orgánico y tradicional). El factor que pudo haber influido en esta valoración corresponde a la amplitud de distancias de siembra usados para facilitar labores culturales como riegos y aplicaciones de agroquímicos.

En lo que se refiere a la calidad del suelo; se puede calificar como suelos de "buena calidad" a los provenientes de los tres agroecosistemas. Esto quiere decir que las condiciones actuales del suelo permitirían continuar con la regularidad de los procesos productivos actuales, al menos en el corto plazo. Sin embargo, se determinaron diferencias en el suelo de cultivo respecto al control (suelo de bosque), en base a los incrementos y/o decrecimientos de parámetros como materia orgánica y nutrientes. Esta valoración expresaría la influencia de las diferentes alternativas tecnológicas en el estado natural del suelo. Los resultados de esta valoración suponen la máxima valoración para los AO y AT y una valoración media para el AC. No obstante, se anota que para evaluar este parámetro en su complejidad se requiere de la implementación de procedimientos que consideren la multiplicidad de variables.

Respecto a la calidad del agua, las fuentes que se utiliza en los ciclos de producción de los AO y AT son consideradas aptas para el uso agropecuario, porque cumplen con los parámetros establecidos en el TULSMA (2003). Cabe mencionar que en el primer caso se trata de agua subterránea y en el segundo caso es agua almacenada proveniente de precipitaciones. El agua utilizada en el AC definitivamente no es apta para el uso agropecuario -al menos no sin un tratamiento- porque supera los niveles permisibles contemplados en el TULSMA (2003), en cuanto a coliformes totales, coliformes fecales y nitratos. Este resultado podría deberse a que el canal de riego recorre grandes distancias antes de llegar al sitio en estudio. En el trayecto recorrido por el canal, el agua es utilizada como abrevadero de animales, para depósito de algunos desechos y para el riego en agricultura bajo la lógica de producción convencional (con agroquímicos).

Para agrobiodiversidad, el AO, según los índices de Margalef $(6,51)$ y de Simpson $(0,90)$ tiene un nivel alto en riqueza y distribución de especies. El AT es también alto en riqueza y distribución de especies con índices de 4,53 y 0,77 para Margalef y Simpson respectivamente. El AC posee un nivel bajo para riqueza en agrobiodiversidad según el índice de Margalef $(0,56)$, sin embargo esta poca riqueza se halla bien distribuida, según el índice de Simpson $(0,83)$. Se observa el efecto de los objetivos de producción que persigue el agricultor, pues cuando se trata del abastecimiento de alimentos para la familia, éste tiende a diversificar la producción. La decisión en la diversificación finalmente influye en la conservación de la productividad del agroecosistema. Ahora, si se trata de la obtención de rentabilidad en el corto plazo, como es el caso del AC la tendencia es hacia seleccionar productos "rentables" y se tiende a uniformizar la producción. 
Un aspecto relevante a mencionar es la riqueza en agrobiodiversidad nativa presente en el AT así como el bagaje de conocimientos que posee la familia propietaria en cuanto a aspectos de comportamiento agronómico, usos, aplicaciones y técnicas para selección, almacenamiento y aprovechamiento. En el AO la riqueza en agrobiodiversidad corresponde a la mixtura de especies nativas y especies introducidas (principalmente hortalizas), mientras que en el AC las especies son principalmente las introducidas, de valor comercial.

La eficiencia energética del sistema, EE es de un nivel medio para el AO, con un valor de 0,22 . Si bien es cierto, en este caso se usan energías alternativas para la producción (energía directa), es el uso de combustibles para la distribución de productos a los consumidores (energía indirecta) el determinante para que la EE del agroecosistema sea considerada de nivel medio. Sin embargo, éste aspecto podría no ser considerado en estudios de este tipo, por cuanto se plantea su cálculo con valores inherentes a la producción, más no a la distribución -que puede ocurrir bajo diversas circunstancias- como lo muestran los ejemplos de cálculos realizados por Risoud (2000). En el AT es de nivel alto con una EE de 0,77 por cuanto no se usa energía no renovable en el ciclo productivo y solo un bajo porcentaje de la producción se destina al mercado (por tanto no se usa mucho combustible). En el AC es bajo con una EE de 0,0030 por el uso de combustibles, plaguicidas y fertilizantes de origen sintético y adquiridos fuera del sistema de producción. Desde el punto de vista energético, este caso evidencia el alto grado de dependencia de insumos externos, y por tanto los altos costos de inversión en el ciclo productivo.

\section{Conclusión}

La cantidad relevante de valores con calificación muy alta en los siete indicadores ambientales a favor de los agroecosistemas orgánico, AO y tradicional, AT, nos llevan a aceptar su contribución desde la dimensión ambiental a la agricultura sustentable. El hecho de que el AC presenta mejor valor únicamente en productividad: biomasa por ha, permite aseverar que el AC no favorece la agricultura sustentable y requiere la urgente corrección en los ámbitos carga tóxica del sistema, residuos de pesticidas en alimentos, agua, suelo, agrobiodiversidad y eficiencia energética, por cuanto es el medio de sustento de la familia.

\section{Agradecimientos}

A las familias campesinas que participaron en este estudio un agradecimiento especial por su colaboración generosa.

\section{Bibliografía}

Agrocalidad (Agencia Ecuatoriana de Aseguramiento de la Calidad del Agro). s/f. "Guía técnica de muestreo de productos agrícolas para la determinación de residuos de plaguicidas (aplicable para vigilancia y control en fincas, empacadoras y mercados)", Anexo General Plan Nacional de Residuos de Contaminantes en productos Primarios, MAGAP, documento de trabajo. Consultado el 20 de julio del 2016, <http://www. agrocalidad.gob.ec/wpcontent/uploads/downloads/2013/10/ANEXO\%20GENERAL\%20PLAN\%20 NACIONAL\%20DE\%20RESIDUOS\%20DE\%20CONTAMINANTES\%20EN\%20PRODUCTOS\%20 PRIMARIOS.pdf>.

Altieri, Miguel. 1999, "Agroecología. Bases científicas para una agricultura sustentable”, editorial NordanComunidad, edición electrónica.

Álvarez, Susana y Bustamante, Teodoro. 2006, «La investigación Agroecológica: ¿Puede contribuir a la disminución de los impactos ambientales?», en: ECUADOR DEBATE, 69:11, en: <http://repositorio. flacsoandes.edu.ec/bitstream/10469/3842/1/RFLACSO-ED69-09-Alvarez.pdf>. 
Comité Ecuménico de Proyectos. 2016, "Organizaciones contrapartes de Pan para el Mundo en Ecuador", Consultado el 10 de junio del 2016, <http://www.cepecuador.org/index.php?option=com_content\&view $=\mathrm{c}$ ategory\&layout $=$ blog $\&$ id $=19 \&$ Itemid $=118 \&$ limitstart $=6>$.

Córdova Vargas, C. y León Sicard, T. 2013, "Resilencia de sistemas agrícolas ecológicos y convencionales frente a la variabilidad climática", en: Agroecología 8 (1), pp. 21-32, en: <https://digitum. um.es/jspui/bitstream/10201/36438/1/Resilencia\%20de\%20sistemas\%20agr\%C3\%ADcolas\%20 ecol $\%$ C $3 \%$ B 3 gicos $\% 20 y \% 20$ convencionales $\% 20$ frente $\% 20$ a $\% 201 \mathrm{a} \% 20$ variabilidad $\% 20$ clim\%C3\%A1tica.pdf>.

FAO y OMS. 2016, CODEX Alimentarius, en: http://www.codexalimentarius.net/mrls/pestdes/pest_ref/ prfs_17.htm>.

Garcés Sandra. 2016, Bienestar y sustentabilidad en el medio rural: análisis de tres agroecosistemas (uno agroecológico, uno convencional y uno mixto) en Carchi y Esmeraldas a través de indicadores multidimensionales, Tesis de maestría, Facultad Latinoamericana de Ciencias Sociales, Sede Ecuador, 2011, en: <http://repositorio.flacsoandes.edu.ec/bitstream/10469/2384/14/TFLACSO-01-SGJ2010.pdf>.

Gliessman, Stephen R. 2002, Agroecología. Procesos Ecológicos en Agricultura Sostenible, CATIE, Costa Rica, edición electrónica.

Hamuda Hosam y István, Patkó. 2010, "Relationship between Environmental Impacts and Modern Agriculture”, en: Óbuda University - Bulletin. Vol. 1, pp. 87 -98, en: <http://uni-obuda.hu/e-bulletin/Hamuda_Patko_1. pdf.>.

Rozano Verónica. 2016, "Hortalizas, Las llaves de la energía”, en: Revista Digital Universitaria. Volumen 5 Número 7, en: <http://www.revista.unam.mx/vol.6/num9/art88/sep_art88.pdf>.

INEN. 2013, "Métodos de muestreo recomendados para la determinación de residuos de plaguicidas a efectos del cumplimiento de los LMR CAC/GL 33-1999". Código de práctica ecuatoriano, 24, CODEX, 2010, Quito, en: <www.fao.org/input/download/standards/361/CXG_033s.pdf >.

INEC. 2013, "Síntesis Metodológica Encuesta de Superficie y Producción Agropecuaria Contínua 2013”, en: $<$ http://www.ecuadorencifras.gob.ec/documentos/web-inec/Estadisticas_agropecuarias/espac/espac\%20 2013/Sintesis_metodologicaESPAC2013.pdf. >.

López, Ridaura Santiago et al. 2001, "Evaluando la sostenibilidad de los sistemas agrícolas integrados: E1 marco MESMIS". BOLETIN DE ILEIA, <http://www.agriculturesnetwork.org/magazines/latin-america/4hacia-la-sostenibilidad-de-los-monocultivos/evaluando-la-sostenibilidad-de-los sistemas/at_download/ article_pdf $>$.

Menalled, Fabián et al. 2008, "An Introduction to the Principles and Practices of Sustainable Farming", en: Agriculture and Natural Resources, en: <http://store.msuextension.org/publications/ AgandNaturalResources/MT200813AG.pdf>.

Moreno, Claudia. 2001, Métodos para medir la biodiversidad, edición electrónica.

Peña-Olvera, Benjamín V. et al. 2008, "Contribución de la dimensión ambiental al desarrollo sustentable de tres agroecosistemas campesinos”, Terra Latinoamerica, 26, pp. 275-284, en: <http://www.redalyc.org/ articulo.oa?id=57313050009>.

Proyecto Andino de Tecnologías Campesinas-PRATEC. 2004, Políticas y Legislación en Agrobiodiversidad, serie Kawsay Mama 6, Lima-Perú, edición electrónica.

Risoud Bernadette. 2000, "Energy efficiency of various French farming systems: questions to sustainability", paper presented at the International Conference Sustainable energy: new challenges for agriculture and implications for land use, Wageningen University, the Netherlands, May 18-20, 2000, en: <https://www2. dijon.inra.fr/cesaer/wp-content/uploads/2012/11/WP2000_9.pdf>.

Ruíz, R. y L.M., Oregui. 2001, "El enfoque sistémico en el análisis de la producción animal: revisión bibliográfica”, Invest. Agr., Prod. Sanid.Animal, Vol. 16 (1), en: <http://www.inia.es/gcontrec/pub/ ruiz_1161096418109.pdf $>$.

Sánchez, Julio et al. 2015, “Agricultura tradicional en El Botho, Alto Mezquital, estado de Hidalgo" en: Revista Mexicana de Ciencias Agricolas 6, pp.1215-1227, en: <http://www.redalyc.org/pdf/2631/Resumenes/ Resumen_263140688006_1.pdf $>$.

Seufert, Verena et al. 2012, "Comparing the yields of organic and conventional agriculture", en: Nature 485 (7397), pp. 229-232. 